\title{
Doxorubicin-loaded human serum albumin nanoparticles overcome transporter-mediated drug resistance in drug-adapted cancer cells
}

\author{
Hannah Onafuye ${ }^{\ddagger 1}$, Sebastian Pieper ${ }^{\ddagger 2}$, Dennis Mulac ${ }^{2}$, Jindrich Cinatl Jr. ${ }^{3}$, \\ Mark N. Wass ${ }^{1}$, Klaus Langer ${ }^{\star 2, \S}$ and Martin Michaelis ${ }^{\star} 1, \uparrow$
}

\section{Full Research Paper}

\section{Address:}

${ }^{1}$ Industrial Biotechnology Centre and School of Biosciences, University of Kent, Canterbury CT2 7NJ, United Kingdom, ${ }^{2}$ Institute of Pharmaceutical Technology and Biopharmacy, University of Münster, Corrensstr. 48, 48149 Münster, Germany and ${ }^{3}$ Institute for Medical Virology, University Hospital, Goethe-University, Paul Ehrlich-Straße 40, 60596 Frankfurt am Main, Germany

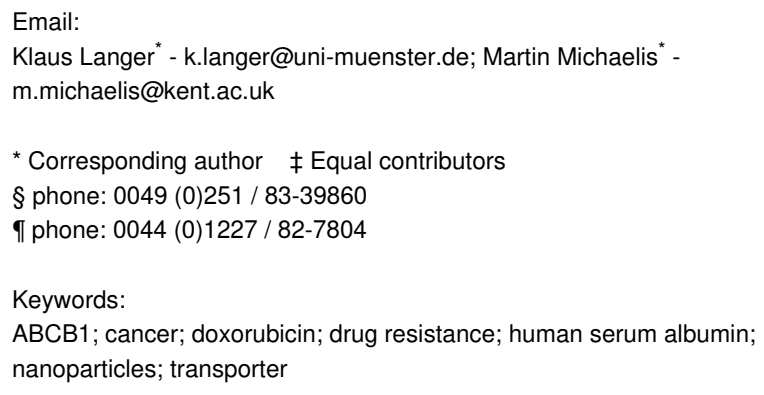

\author{
Beilstein J. Nanotechnol. 2019, 10, 1707-1715. \\ doi:10.3762/bjnano.10.166
}

Received: 30 May 2019

Accepted: 29 July 2019

Published: 14 August 2019

This article is part of the thematic issue "Frontiers in pharmaceutical nanotechnology".

Guest Editor: M. G. Wacker

(C) 2019 Onafuye et al.; licensee Beilstein-Institut. License and terms: see end of document.

\begin{abstract}
Resistance to systemic drug therapy is a major reason for the failure of anticancer therapies. Here, we tested doxorubicin-loaded human serum albumin (HSA) nanoparticles in the neuroblastoma cell line UKF-NB-3 and its ABCB1-expressing sublines adapted to vincristine (UKF-NB- $3^{\mathrm{r}} \mathrm{VCR}^{1}$ ) and doxorubicin (UKF-NB-3 ${ }^{\mathrm{r}} \mathrm{DOX}{ }^{20}$ ). Doxorubicin-loaded nanoparticles displayed increased anticancer activity in UKF-NB- $3^{\mathrm{r}} \mathrm{VCR}^{1}$ and UKF-NB- $3^{\mathrm{r}} \mathrm{DOX}{ }^{20}$ cells relative to doxorubicin solution, but not in UKF-NB-3 cells. UKF-NB- $3^{r} V_{C R}{ }^{1}$ cells were re-sensitised by nanoparticle-encapsulated doxorubicin to the level of UKF-NB-3 cells. UKF-NB$3^{\mathrm{r}} \mathrm{DOX}{ }^{20}$ cells displayed a more pronounced resistance phenotype than UKF-NB- $3^{\mathrm{r}} \mathrm{VCR}^{1}$ cells and were not re-sensitised by doxorubicin-loaded nanoparticles to the level of parental cells. ABCB1 inhibition using zosuquidar resulted in similar effects like nanoparticle incorporation, indicating that doxorubicin-loaded nanoparticles successfully circumvent ABCB1-mediated drug efflux. The limited re-sensitisation of UKF-NB- $3^{\mathrm{r}} \mathrm{DOX}{ }^{20}$ cells to doxorubicin by circumvention of ABCB1-mediated efflux is probably due to the presence of multiple doxorubicin resistance mechanisms. So far, ABCB1 inhibitors have failed in clinical trials probably because systemic $\mathrm{ABCB} 1$ inhibition results in a modified body distribution of its many substrates including drugs, xenobiotics, and other molecules. HSA nanoparticles may provide an alternative, more specific way to overcome transporter-mediated resistance.
\end{abstract}




\section{Introduction}

According to Globocan there "were 14.1 million new cancer cases, 8.2 million cancer deaths and 32.6 million people living with cancer (within 5 years of diagnosis) in 2012 worldwide" [1]. Despite substantial improvements over recent decades, the prognosis for many cancer patients remains unacceptably poor. The outlook is particularly grim for patients that are diagnosed with disseminated (metastatic) disease who cannot be successfully treated by local treatment (surgery, radiotherapy) and depend on systemic drug therapy, because the success of systemic therapies is typically limited by therapy resistance [2-4].

Drug efflux mediated by transporters including adenosine triphosphate (ATP)-binding cassette (ABC) transporters has been shown to play a crucial role in cancer cell drug resistance [2,5]. ABCB1 (also known as P-glycoprotein or MDR1) seems to play a particularly important role in cancer cell drug resistance as a highly promiscuous transporter that mediates the cellular efflux of a wide range of structurally different substrates including many anticancer drugs. Different studies have reported that nanometer-sized drug carrier systems can bypass efflux-mediated drug resistance [6]. This includes various nanoparticle and liposome formulations of the ABCB1 substrate doxorubicin [7-12].

Here, we here investigated the effects of doxorubicin-loaded human serum albumin (HSA) nanoparticles in ABCB1expressing neuroblastoma cells. HSA nanoparticles are easy to produce [13-17], and HSA is a well-tolerated material. It is the most abundant protein in human blood plasma and used in many pharmaceutical formulations, in particular as part of critical care treatment [18].

\section{Results}

\section{Nanoparticle size, polydispersity and drug load}

HSA nanoparticles were prepared by desolvation as previously described [13-17]. The nanoparticles were stabilised by the cross-linking of free amino groups present in albumin. Three different nanoparticle preparations were produced using glutaraldehyde at amounts that corresponded to a theoretical cross-linking of 40\% (HSA 40\% nanoparticles), 100\% (HSA
$100 \%$ nanoparticles), or $200 \%$ (HSA $200 \%$ nanoparticles) of the amino groups that are available in the HSA molecules. A nonstabilised ( $0 \%$ cross-linking) formulation was used as a control. The resulting particle sizes and polydispersity indices are shown in Table 1. HSA ( $0 \%$ ) nanoparticles displayed a large particle size of almost $1 \mu \mathrm{m}$ and a high polydispersity of 0.5 , confirming that no stable nanoparticles had formed (Table 1). The three HSA nanoparticle preparations stabilised by the different glutaraldehyde concentrations displayed similar diameters between 460 and $500 \mathrm{~nm}$ and polydispersity indices in the range of 0.153 and 0.213 , indicating a narrow but not monodisperse size distribution (Table 1).

The spherical shape and narrow size distribution of HSA nanoparticles was confirmed by scanning electron microscopy (SEM) as depicted for nanoparticles stabilised by a $100 \%$ crosslinking degree (Figure 1). For these nanoparticles a zeta potential of $-12.5 \pm 1.8 \mathrm{mV}(n=6)$ was detected, indicating only a moderate stabilisation by electrostatic repulsion.

While HSA (40\%), HSA (100\%), and HSA (200\%) nanoparticles displayed similar drug loads between 152 and $191 \mu \mathrm{g}$ doxorubicin/mg nanoparticle, HSA (0\%) nanoparticles had bound $371 \mu \mathrm{g}$ doxorubicin/mg HSA (Table 1). This probably reflected the higher accessibility of doxorubicin binding sites, which are known to be available on HSA [19], in HSA molecules in solution compared to the accessible binding sites available in HSA nanoparticles.

\section{Doxorubicin sensitivity of the used neuroblastoma cell lines}

The parental neuroblastoma cell line UKF-NB-3 and its doxorubicin- $\left(\mathrm{UKF}-\mathrm{NB}-3^{\mathrm{r}} \mathrm{DOX}^{20}\right.$ ) and vincristine-adapted (UKF-NB$3^{\mathrm{r}} \mathrm{VCR}^{1}$ ) sub-lines substantially differed in their doxorubicin sensitivity (Figure 2). UKF-NB-3 displayed the lowest doxorubicin $\mathrm{IC}_{50}(3.8 \mathrm{ng} / \mathrm{mL})$. UKF-NB- $3^{\mathrm{r}} \mathrm{VCR}^{1}$ was 4 -fold more resistant to doxorubicin than UKF-NB-3 (doxorubicin $\mathrm{IC}_{50}$ : $15.5 \mathrm{ng} / \mathrm{mL}$ ). UKF-NB- $3^{\mathrm{r}} \mathrm{DOX}^{20}$ showed the highest doxorubicin $\mathrm{IC}_{50}(89.0 \mathrm{ng} / \mathrm{mL})$, resulting in a 23 -fold increase in doxorubicin resistance compared to UKF-NB-3 (Figure 2, Supporting Information File 1, Table S1).

Table 1: Nanoparticle diameter, polydispersity, and drug load.

\begin{tabular}{llll} 
Nanoparticle formulations & Diameter $(\mathrm{nm})$ & Polydispersity & Drug load $(\mu \mathrm{g}$ doxorubicin/mg nanoparticle) \\
\hline HSA (0\%) & 848.7 & 0.500 & 370.9 \\
HSA (40\%) & 485.8 & 0.189 & 151.9 \\
HSA (100\%) & 496.4 & 0.213 & 190.5 \\
HSA (200\%) & 463.4 & 0.153 & 164.8
\end{tabular}




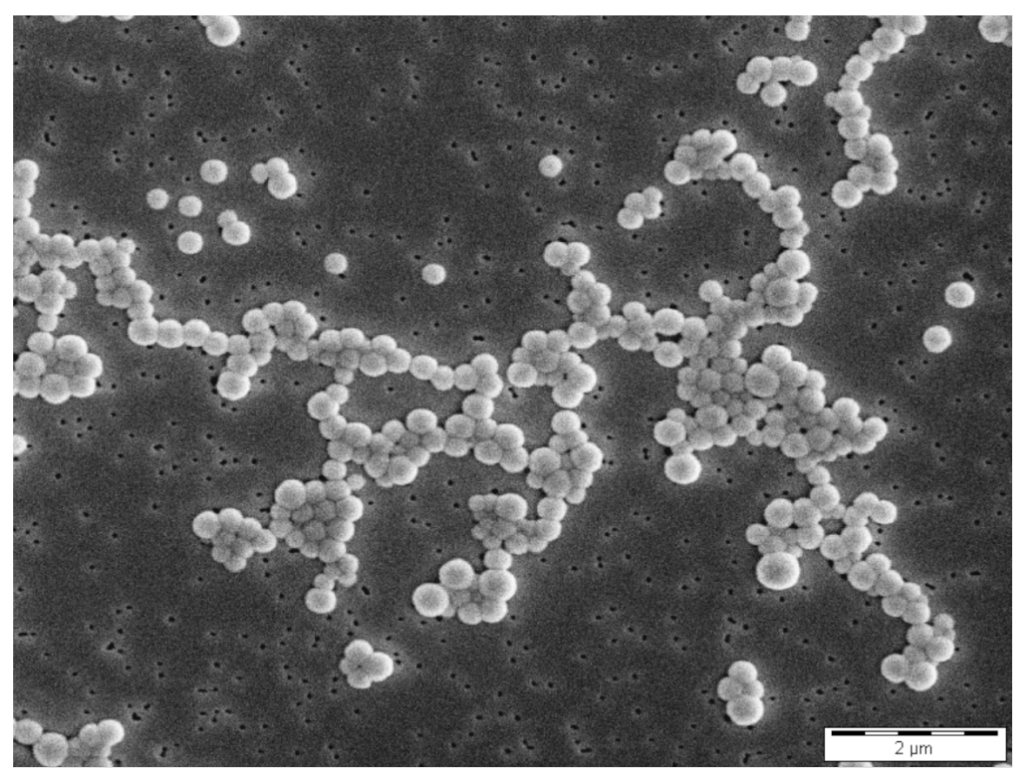

Figure 1: SEM confirmed the spherical shape and narrow size distribution of doxorubicin-loaded HSA (100\%) nanoparticles.
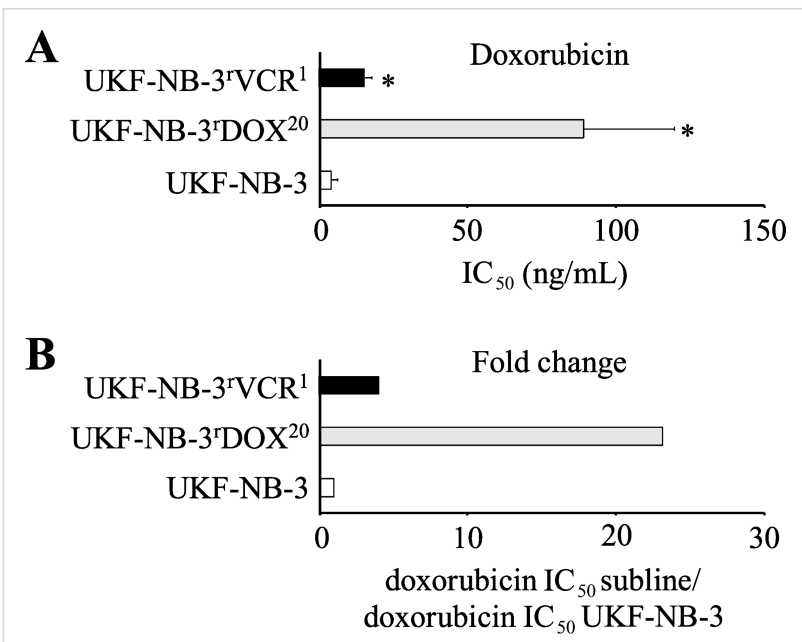

Figure 2: Doxorubicin sensitivity of UKF-NB-3, its doxorubicin-adapted sub-line UKF-NB-3'DOX ${ }^{20}$ and its vincristine-adapted sub-line UKFNB-3rVCR ${ }^{1}$. A) Doxorubicin concentrations that reduce cell viability by $50 \%\left(\mathrm{IC}_{50}\right)$ as indicated by MTT assay after $120 \mathrm{~h}$ of incubation. B) Fold change in doxorubicin sensitivity (doxorubicin IC 50 UKF-NB-3 sub-line/doxorubicin $\mathrm{IC}_{50}$ UKF-NB-3). Numerical values are presented in Supporting Information File 1, Table $\mathrm{S} 1 .{ }^{*} P<0.05$ relative to UKFNB-3.

\section{Effects of doxorubicin-loaded nanoparticles on neuroblastoma cells}

The effects of doxorubicin applied in solution or incorporated into HSA $(0 \%)$, HSA $(40 \%)$, HSA $(100 \%)$, or HSA $(200 \%)$ nanoparticles on neuroblastoma cell viability are shown in Figure 3. The numerical values are presented in Supporting Information File 1, Table S1. Empty control nanoparticles did not affect cell viability in the investigated concentrations.
In the neuroblastoma cell line UKF-NB-3, the nanoparticle preparations displayed similar activity as doxorubicin solution, with doxorubicin-loaded HSA (40\%), HSA (100\%), and HSA (200\%) nanoparticles potentially showing a trend towards a slightly increased activity (Figure 3). However, the differences did not reach statistical significance. Similar results were obtained in the doxorubicin-adapted UKF-NB-3 sub-line UKF$\mathrm{NB}-3^{\mathrm{r}} \mathrm{DOX}{ }^{20}$, although the difference between doxorubicinloaded HSA (200\%) nanoparticles and doxorubicin solution reached statistical significance (Figure 3). Notably, nonstabilised doxorubicin-bound HSA ( $0 \%)$ nanoparticles differed in their relative activity and did not reduce UKF-NB- $3^{\mathrm{r}} \mathrm{DOX}{ }^{20}$ viability by $50 \%$ within the observed concentration range up to $200 \mathrm{ng} / \mathrm{mL}$.

The vincristine-adapted UKF-NB-3 sub-line UKF-NB-3rVCR1 displayed decreased doxorubicin sensitivity. However, doxorubicin-loaded HSA (40\%), HSA (100\%), and HSA (200\%) nanoparticles displayed a higher relative potency compared to doxorubicin solution in UKF-NB- $3^{\mathrm{r}} \mathrm{VCR}^{1}$ (Figure 3, Figure 4). The fold sensitisation doxorubicin $\mathrm{IC}_{50}$ doxorubicin solution/ doxorubicin $\mathrm{IC}_{50}$ nanoparticle-bound doxorubicin for HSA (40\%), HSA $(100 \%)$, and HSA $(200 \%)$ nanoparticles (3.6-4.5-fold) was higher than for UKF-NB-3 (1.9-2.5-fold), and UKF-NB- $3{ }^{\mathrm{r}} \mathrm{DOX}{ }^{20}$ (2.1-2.9-fold). The differences between doxorubicin-loaded HSA (40\%) nanoparticles, HSA $(100 \%)$ nanoparticles, and HSA (200\%) nanoparticles and doxorubicin solution reached statistical significance $(P<0.05)$ (Figure 3). Doxorubicin encapsulation into HSA (40\%), HSA $(100 \%)$, or HSA $(200 \%)$ nanoparticles reduced the doxorubicin 

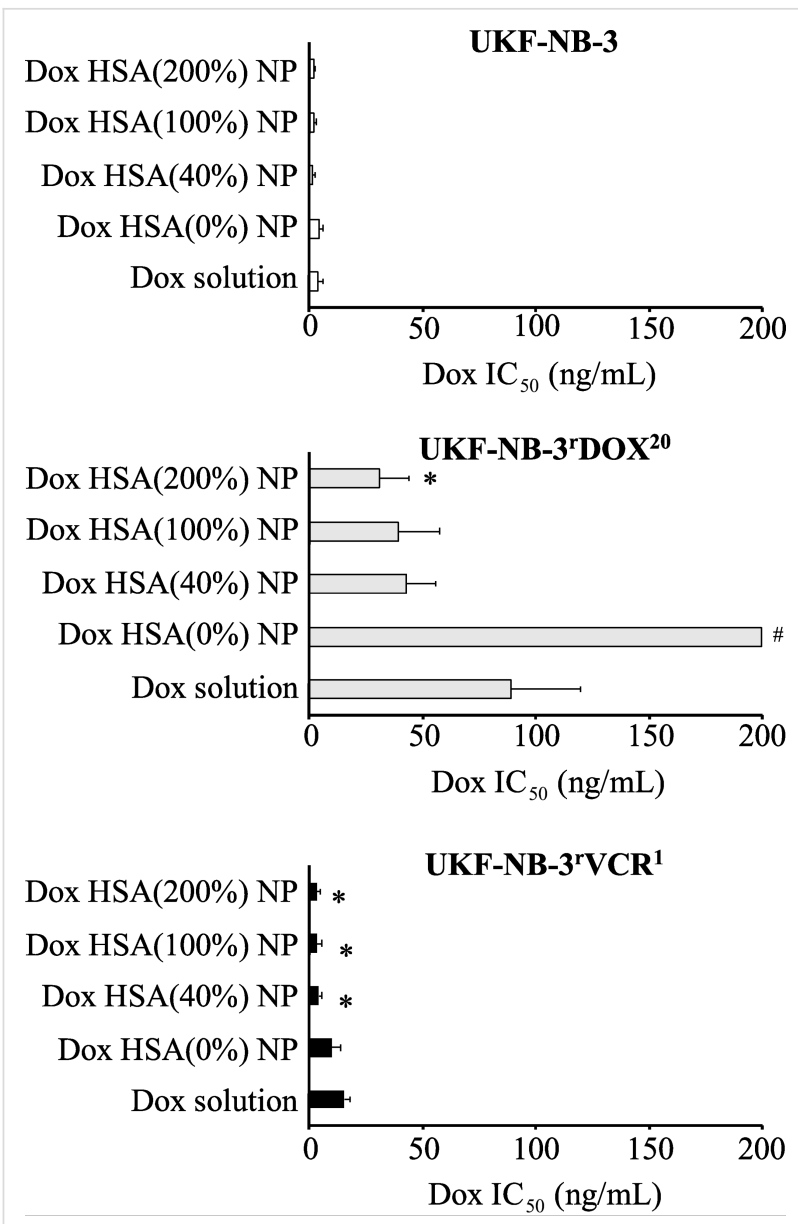

Figure 3: Effects of doxorubicin (Dox) applied as a solution or incorporated into human serum albumin (HSA) nanoparticles on neuroblastoma cell viability. The investigated nanoparticles differed in the amount of the cross-linker glutaraldehyde that was used for nanoparticle stabilisation. The amount of glutaraldehyde corresponded to $40 \%$ (Dox HSA (40\%) NP), 100\% (Dox HSA (100\%) NP), or 200\% (Dox HSA (200\%) NP) theoretical cross-linking of the available amino groups present on HSA. Preparations prepared without glutaraldehyde served as a control (Dox HSA ( $0 \%$ ) NP). Values are expressed as concentrations that reduce cell viability by $50 \%\left(\mathrm{IC}_{50}\right)$ as determined by MTT assay after $120 \mathrm{~h}$ of incubation. Numerical values are presented in Supporting Information File 1, Table S1. Empty nanoparticles did not affect cell viability in the investigated concentrations. ${ }^{*} P<0.05$ relative to doxorubicin solution; ${ }^{\#} \mathrm{IC}_{50}>200 \mathrm{ng} / \mathrm{mL}$.

$\mathrm{IC}_{50}$ in UKF-NB- $3^{\mathrm{r}} \mathrm{VCR}^{1}$ cells to the levels of doxorubicin solution in parental UKF-NB-3 cells (Figure 3, Supporting Information File 1, Table S1). In contrast, the doxorubicin $\mathrm{IC}_{50}$ of doxorubicin-loaded HSA nanoparticles remained clearly (8-11-fold) higher in UKF-NB-3 ${ }^{\mathrm{r}}$ DOX ${ }^{20}$ cells than the doxorubicin $\mathrm{IC}_{50}$ of doxorubicin solution in parental UKF-NB-3 cells.

\section{Effects of the ABCB1 inhibitor zosuquidar on the efficacy of nanoparticle-bound doxorubicin in UKF-NB-3r ${ }^{r} O^{20}$ cells}

Doxorubicin is an ABCB1 substrate, and UKF-NB- $3^{\mathrm{r}} \mathrm{DOX}^{20}$ cells are characterised by high ABCB1 expression [20,21].
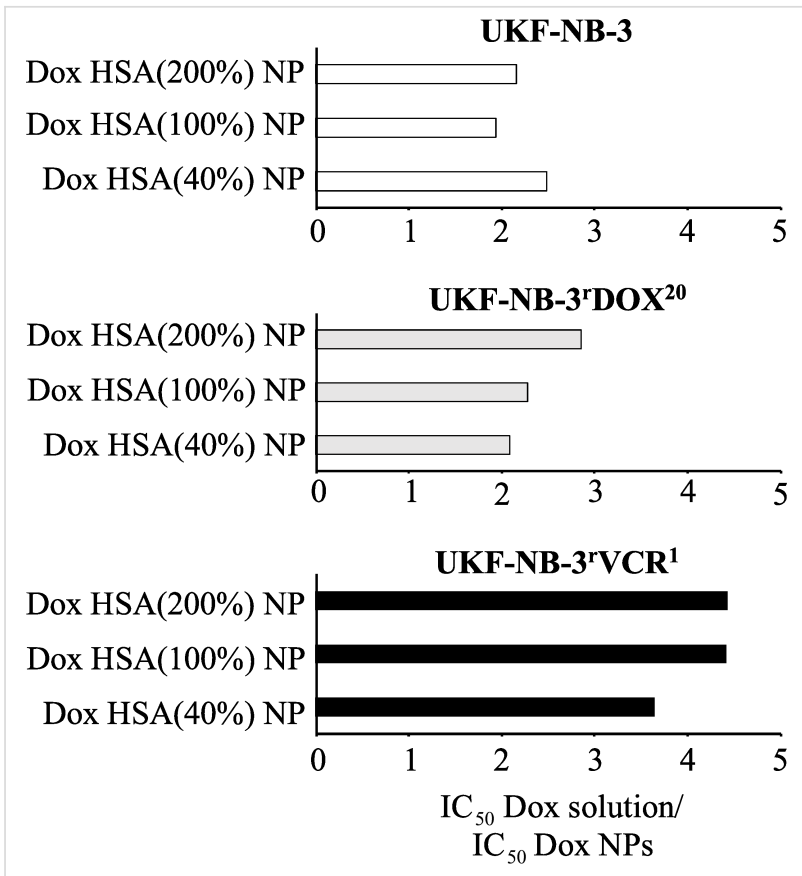

Figure 4: Fold sensitisation to doxorubicin by doxorubicin-bound nanoparticles (NPs). Values are expressed as fold changes doxorubicin (Dox) $\mathrm{IC}_{50}$ of doxorubicin solution/doxorubicin $\mathrm{IC}_{50}$ of doxorubicinbound NPs. Human serum albumin (HSA) nanoparticles were stabilised by glutaraldehyde concentrations corresponding to $40 \%$ (Dox HSA (40\%) NP), 100\% (Dox HSA (100\%) NP), or 200\% (Dox HSA (200\%) NP) theoretical cross-linking of the available amino groups present on HSA.

Vincristine is also an ABCB1 substrate, and vincristine-adapted cancer cell lines often display enhanced ABCB1 levels $[20,22,23]$. Accordingly, UKF-NB- $3^{r} V_{C R}{ }^{1}$ cells are sensitised by the ABCB1 inhibitor zosuquidar [2-6] to doxorubicin to the level of parental UKF-NB-3 cells (Supporting Information File 1, Figure S1), which indicates that ABCB1 expression contributes to the resistance phenotype observed in UKF-NB$3^{\mathrm{r}} \mathrm{VCR}^{1}$ cells.

Doxorubicin bound to nanometer-sized drug carrier systems has been shown to bypass ABCB1-mediated drug efflux [7-12]. In UKF-NB- $3^{\mathrm{r}} \mathrm{VCR}^{1}$ cells, combining both doxorubicin with zosuquidar and doxorubicin encapsulation into HSA nanoparticles reduced the doxorubicin $\mathrm{IC}_{50}$ to the level of parental UKFNB-3 cells (Figure 3, Supporting Information File 1, Figure S1, Table S1), which do not display detectable ABCB1 activity $[20,22,23]$. Hence, the increased activity of nanoparticle-bound doxorubicin that we observed in UKF-NB- $3^{r} V_{C R}{ }^{10}$ cells is likely to be attributed to the circumvention of ABCB1-mediated doxorubicin efflux.

In UKF-NB- $3^{\mathrm{r}}$ DOX ${ }^{20}$ cells, however, the differences between doxorubicin solution and doxorubicin nanoparticles only reached statistical significance for doxorubicin-loaded HSA 
(200\%) nanoparticles (Figure 3). The reasons for this may include that nanoparticle-incorporated doxorubicin does not completely avoid ABCB1-mediated efflux from UKF-NB$3^{\mathrm{r}} \mathrm{DOX}^{20}$ cells and/or that doxorubicin resistance is caused by multiple resistance mechanisms and that avoidance of ABCB1mediated transport is not sufficient to re-sensitise UKF-NB$3^{\mathrm{r}} \mathrm{DOX}{ }^{20}$ cells to doxorubicin to the level of UKF-NB-3 cells.

To further study the role of $\mathrm{ABCB} 1$ as a doxorubicin resistance mechanism in UKF-NB-3 $3^{\mathrm{r}} \mathrm{DOX}^{20}$ cells, we performed additional experiments in which we combined the ABCB1 inhibitor zosuquidar and doxorubicin applied as a solution or nanoparticle preparations in UKF-NB- $3^{\mathrm{r}} \mathrm{DOX}{ }^{20}$ and UKF-NB-3 cells. Zosuquidar $(1 \mu \mathrm{M})$ did not affect the efficacy of doxorubicin solution or nanoparticle-bound doxorubicin in parental UKFNB-3 cells (Figure 5), which do not display noticeable ABCB1 activity $[20,22,23]$. These experiments also confirmed that there is no significant difference in the anticancer activity between doxorubicin solution and doxorubicin nanoparticles in UKFNB-3 cells, despite an apparent trend in the first set of experiments (Figure 3).

In UKF-NB-3 $3^{\mathrm{r} D O X^{20}}$ cells, the addition of zosuquidar resulted in an increased sensitivity to free doxorubicin (Figure 5). The doxorubicin $\mathrm{IC}_{50}$ decreased by 2.5 -fold from $91 \mathrm{ng} / \mathrm{mL}$ in the absence of zosuquidar to $37 \mathrm{ng} / \mathrm{mL}$ in the presence of zosuquidar, but not to the level of UKF-NB-3 cells $(4.6 \mathrm{ng} / \mathrm{mL})$ (Supporting Information File 1, Table S2). This confirmed that $\mathrm{ABCB} 1$ is one among multiple resistance mechanisms that contribute to the doxorubicin resistance phenotype observed in UKF-NB- $3^{\mathrm{r}}{ }^{\mathrm{DOX}}{ }^{20}$.

In this set of experiments, doxorubicin-loaded nanoparticles displayed a significantly increased activity compared to doxorubicin solution in UKF-NB- $3^{\mathrm{r}} \mathrm{DOX}{ }^{20}$ cells (Figure 5). This finding together with the non-significant trend observed in the first set of experiments (Figure 3) suggests that doxorubicinloaded nanoparticles do indeed exert stronger effects against UKF-NB-3 ${ }^{\mathrm{r}} \mathrm{DOX}{ }^{20}$ cells than doxorubicin solution. Zosuquidar only moderately increased the efficacy of doxorubicin nanoparticles further (1.1-1.8-fold) in UKF-NB- $3^{\mathrm{r} D O X^{20}}$ cells (Figure 5, Supporting Information File 1, Table S2). In particular, the anticancer effects of doxorubicin-loaded HSA (200\%) nanoparticles, the most active nanoparticle preparation in UKFNB-3 $3^{\mathrm{r}}$ DOX ${ }^{20}$ cells, displayed a doxorubicin $\mathrm{IC}_{50}$ of $20 \mathrm{ng} / \mathrm{mL}$, which was not further reduced by addition of zosuquidar (doxorubicin $\mathrm{IC}_{50}: 18 \mathrm{ng} / \mathrm{mL}$ ) (Figure 5, Table S2). Hence, the increased anticancer activity of doxorubicin incorporated into HSA nanoparticles appears to be primarily caused by circumventing the ABCB1-mediated doxorubicin efflux in UKF-NB$3^{\mathrm{r}} \mathrm{DOX}^{20}$ cells.

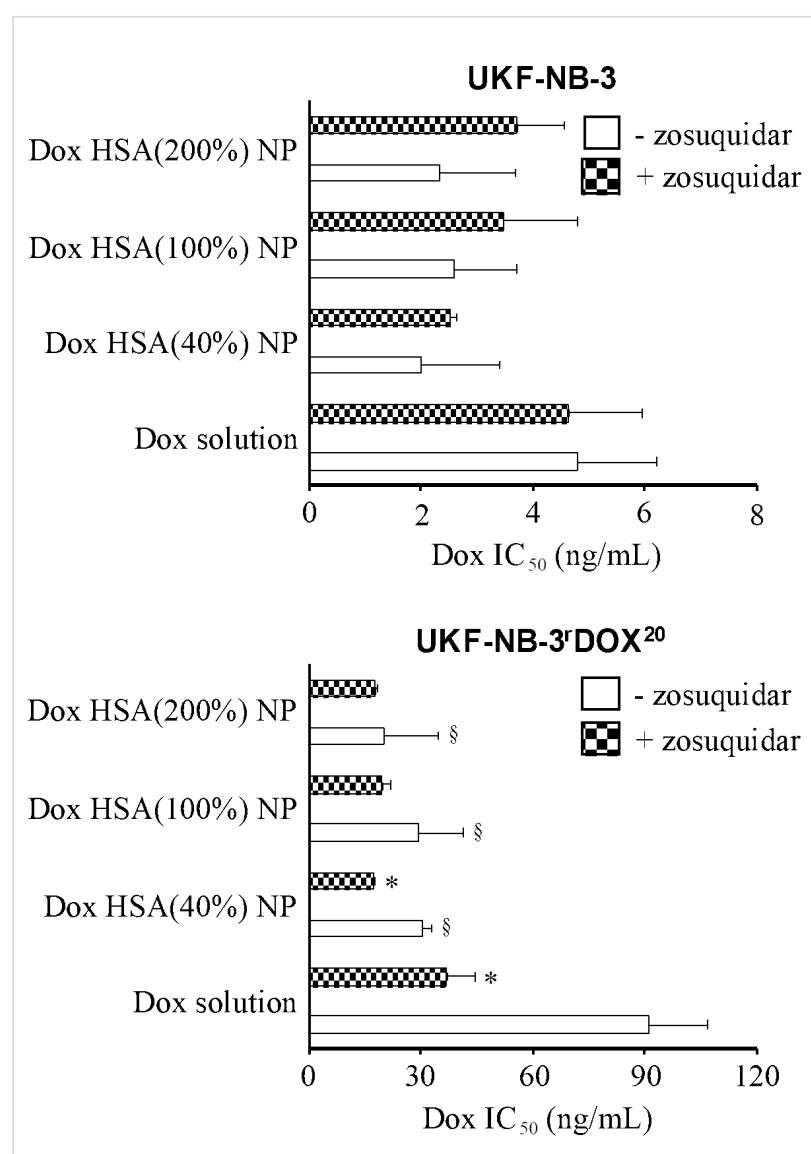

Figure 5: Doxorubicin (Dox) concentrations that reduce neuroblastoma cell viability by $50 \%\left(\mathrm{IC}_{50}\right)$ in the presence or absence of the ABCB1 inhibitor zosuquidar $(1 \mu \mathrm{M})$ as determined by MTT assay after $120 \mathrm{~h}$ incubation. Doxorubicin was either applied as a solution or incorporated into human serum albumin (HSA) nanoparticles which had been stabilised by addition of glutaraldehyde concentrations corresponding to $40 \%$ (Dox HSA (40\%) NP), $100 \%$ (Dox HSA (100\%) NP), or $200 \%$ (Dox HSA (200\%) NP) theoretical cross-linking of the available amino groups present on HSA. Zosuquidar $(1 \mu \mathrm{M})$ did not affect cell viability on its own. Numerical data are presented in Supporting Information File 1 , Table S2. ${ }^{*} P<0.05$ relative to the doxorubicin $\mathrm{IC}_{50}$ in the absence of zosuquidar; $\S P<0.05$ relative to doxorubicin solution.

\section{Discussion}

The occurrence of drug resistance is the major reason for the failure of systemic anticancer therapies [2]. Here, we investigated the effects of doxorubicin-loaded HSA nanoparticles on the viability of the neuroblastoma cell line UKF-NB-3 and its sublines adapted to doxorubicin (UKF-NB- ${ }^{\mathrm{r}} \mathrm{DOX}{ }^{20}$ ) and vincristine (UKF-NB- $3^{\mathrm{r}} \mathrm{VCR}^{1}$ ), which both display ABCB1 activity and resistance to doxorubicin. The HSA nanoparticles were prepared by desolvation and stabilised by glutaraldehyde, which crosslinks amino groups present in albumin molecules [13-17]. Glutaraldehyde was used at molar concentrations that corresponded to $40 \%$ (Dox HSA (40\%) nanoparticles), 100\% (Dox HSA (100\%) nanoparticles), or 200\% (Dox HSA (200\%) nanoparticles) theoretical cross-linking of the 59 amino groups 
available per HSA molecule [24]. The resulting nanoparticles ranged from 463 to $486 \mathrm{~nm}$ in diameter and had a low polydispersity index in the range of 0.2 .

Doxorubicin-loaded nanoparticles displayed similar activity as doxorubicin solution in the parental UKF-NB-3 cell line, but exerted stronger effects than doxorubicin solution in the ABCB1-expressing UKF-NB-3 sub-lines. The UKF-NB$3^{\mathrm{r}} \mathrm{VCR}^{1}$ cells were similarly sensitive to doxorubicin-loaded nanoparticles as parental UKF-NB-3 cells to doxorubicin solution (and doxorubicin-loaded nanoparticles). This suggests that the doxorubicin resistance of UKF-NB- $3^{r} V R^{1}$ cells exclusively depends on $\mathrm{ABCB} 1$ expression. In concordance, the $\mathrm{ABCB} 1$ inhibitor zosuquidar re-sensitised UKF-NB- $3^{\mathrm{r}} \mathrm{VCR}^{1}$ cells to the level of parental UKF-NB-3 cells.

The UKF-NB- $3^{\mathrm{r}} \mathrm{DOX}{ }^{20}$ cells displayed a more pronounced doxorubicin resistance phenotype than UKF-NB- $3^{\mathrm{r}} \mathrm{VCR}^{1}$ cells and were neither re-sensitised by nanoparticle-encapsulated doxorubicin nor by zosuquidar to the level of UKF-NB-3 cells. This suggests that UKF-NB- $3^{\mathrm{r}} \mathrm{DOX}{ }^{20}$ cells have developed multiple doxorubicin resistance mechanisms. In contrast, adaptation of UKF-NB- $3^{\mathrm{r}} \mathrm{VCR}^{1}$ cells to vincristine, a tubulinbinding agent with an anticancer mechanism of action that is not related to that of the topoisomerase II inhibitor doxorubicin $[2,20,25,26]$, did not result in the acquisition of changes that confer doxorubicin resistance beyond $\mathrm{ABCB} 1$ expression.

Furthermore, zosuquidar did not increase the efficacy of doxorubicin-loaded HSA (100\%) and HSA (200\%) nanoparticles and only modestly enhanced the efficacy of doxorubicinloaded HSA (40\%) nanoparticles. Together, these data confirm that administration of doxorubicin as HSA nanoparticles resulted in the circumvention of ABCB1-mediated drug efflux. The difference between HSA (40\%) nanoparticles and the other two preparations may be explained by elevated drug release due to the lower degree of cross-linking.

Interestingly, high concentrations of the cross-linker glutaraldehyde did not affect the efficacy of the resulting doxorubicinloaded nanoparticles although high glutaraldehyde concentrations might have been expected to affect drug release and/or to covalently bind to doxorubicin via its amino group.

Notably, the results differ from a recent similar study in which nanoparticles prepared from poly(lactic-co-glycolic acid) (PLGA) or polylactic acid (PLA), two other biodegradable materials approved by the FDA and EMA for human use [27,28], did not bypass ABCB1-mediated drug efflux [29]. Differences in the mode of uptake and cellular distribution of the nanoparticles from different materials may be responsible for these discrepancies. HSA nanoparticles may be internalised upon interaction with cellular albumin receptors [30,31]. Notably, nab-paclitaxel, an HSA nanoparticle-based preparation of paclitaxel (another ABCB1 substrate [21]), which is approved for the treatment of different forms of cancer [32], had previously been shown not to avoid ABCB1-mediated drug efflux [33]. However, nab-paclitaxel is not produced by the use of crosslinkers, and the interaction of paclitaxel with albumin may differ from that of doxorubicin. Hence, variations in drug binding and drug release kinetics may be responsible for this difference.

Despite the prominent role of $\mathrm{ABCB} 1$ as a drug resistance mechanism, attempts to exploit it as drug target have failed so far, despite the development of highly specific allosteric $\mathrm{ABCB} 1$ inhibitors (of which zosuquidar is one) [5,21]. One reason for this is that $\mathrm{ABCB} 1$ is expressed at various physiological borders and involved in the control of the body distribution of its many endogenous and exogenous substrates. Systemic ABCB1 inhibition can therefore result in toxicity as a consequence of a modified body distribution of anticancer drugs (and other drugs that are co-administered for conditions other than cancer), xenobiotics, and other molecules. Hence, the use of drug carrier systems to bypass $\mathrm{ABC}$ transporter-mediated drug efflux is conceptually very attractive because it can (in contrast to inhibitors of $\mathrm{ABCB} 1$ or other transporters) overcome resistance mediated by multiple transporters and does not result in the systemic inhibition of transporter function at physiological barriers. However, cancer cells may be characterised by multiple further resistance mechanisms and just bypassing transporter-mediated efflux may not be sufficient to achieve therapeutic response (as illustrated by our current finding that UKF-NB- $3{ }^{\mathrm{r}} \mathrm{DOX}{ }^{20}$ cells cannot be fully re-sensitised to doxorubicin by zosuquidar) $[2,5,21]$. Hence, our results demonstrate that more sophisticated, personalised therapies will need to be developed. Such therapies will depend on an improved understanding of the resistance status of cancer cells to a certain drug beyond its transporter status. If biomarkers become available that predict cancer cell response to a certain drug more reliably, nanoparticles can be used to transport drugs under circumvention of transporter-mediated efflux into cancer cells that are likely to respond to them.

In conclusion, doxorubicin-loaded HSA nanoparticles produced by desolvation and cross-linking using glutaraldehyde overcome (in contrast to other nanoparticle systems) transporter-mediated drug resistance in drug-adapted neuroblastoma cells. However, our data also show that bypassing of transporter-mediated drug efflux may not be sufficient to sensitise cancer cells, which have developed multiple resistance mechanisms, to the level of sensitive parental cells. 


\section{Experimental}

\section{Reagents and chemicals}

HSA and glutaraldehyde were obtained from Sigma-Aldrich Chemie GmbH (Karlsruhe, Germany). Dulbecco's phosphate buffered saline (PBS) was purchased from Biochrom $\mathrm{GmbH}$ (Berlin, Germany). Doxorubicin was obtained from LGC Standards GmbH (Wesel, Germany). All chemicals were of analytical grade and used as received.

\section{Human serum albumin (HSA) nanoparticle preparation by desolvation}

HSA nanoparticles were prepared by desolvation as previously described [13-17]. $100 \mu \mathrm{L}$ of a $1 \%(\mathrm{w} / \mathrm{v})$ aqueous doxorubicin solution was added to $500 \mu \mathrm{L}$ of a $40 \mathrm{mg} / \mathrm{mL}$ (w/v) HSA solution and incubated for $2 \mathrm{~h}$ at room temperature under stirring (550 rpm, Cimaric i Multipoint Stirrer, ThermoFisher Scientific, Langenselbold, Germany). $4 \mathrm{~mL}$ of ethanol $96 \%$ was added at room temperature under stirring using a peristaltic pump (Ismatec ecoline, Ismatec, Wertheim-Mondfeld, Germany) at a flow rate of $1 \mathrm{~mL} / \mathrm{min}$. After the desolvation process, the resulting nanoparticles were stabilised/cross-linked using different amounts of glutaraldehyde that corresponded to different percentages of the theoretical amount that is necessary for the quantitative cross-linking of the 60 primary amino groups present in the HSA molecules of the particle matrix. The addition of $4.7 \mu \mathrm{L} 8 \%(\mathrm{w} / \mathrm{v})$ aqueous glutaraldehyde solution resulted in a theoretical cross-linking of $40 \%$ of the HSA amino groups, the addition of $11.8 \mu \mathrm{L} 8 \%(w / v)$ aqueous glutaraldehyde solution in $100 \%$ cross-linking, and the addition of $23.6 \mu \mathrm{L} 8 \%(\mathrm{w} / \mathrm{v})$ aqueous glutaraldehyde solution in $200 \%$ cross-linking. The suspension was then stirred for $12 \mathrm{~h}$ at $550 \mathrm{rpm}$. The particles were purified by centrifugation (at $16,000 \mathrm{~g}$ for $12 \mathrm{~min}$ ) and resuspension steps performed three times in purified water. During the particle purification the supernatants were collected and the drug content was measured by high-performance liquid chromatography (HPLC) as described below. The loading efficiency of doxorubicin in the nanoparticles was calculated based on the difference between the doxorubicin amount used for nanoparticle preparation and the unbound amount detected in the collected supernatants.

\section{Determination of particle size distribution}

The average particle size and the polydispersity were measured by photon correlation spectroscopy (PCS) using a Malvern zetasizer nano instrument (Malvern Instruments, Herrenberg, Germany). The resulting particle suspensions were diluted 1:100 with purified water and measured at a temperature of $22{ }^{\circ} \mathrm{C}$ using a backscattering angle of $173^{\circ}$.

The zeta potential was measured in the same instrument by laser Doppler microelectrophoresis to provide information about the surface charge of the nanoparticles. Thus, the nanoparticle dilutions described above were transferred into a folded capillary cell and the experiment was conducted at $22^{\circ} \mathrm{C}$.

\section{Morphological analysis of nanoparticles by scanning electron microscopy (SEM)}

$3 \mu \mathrm{L}$ of diluted HAS nanoparticle suspension $(0.25 \mathrm{mg} / \mathrm{mL})$ was applied on a $0.1 \mu \mathrm{m}$ membrane filter (Isopore ${ }^{\mathrm{TM}}$ membrane filter, Merck Millipore, Darmstadt, Germany) and dried overnight in a desiccator. Afterwards, the membrane filter was sputtered with gold (Sputter SCD 040, BALTEC, Liechtenstein) under argon atmosphere. SEM was performed on a CamScan CS4 microscope (Cambridge Scanning Company, Cambridge, United Kingdom) and the sample was visualised with an accelerating voltage of $10 \mathrm{kV}$, a working distance of $10 \mathrm{~mm}$, and 10,000-fold magnification.

\section{Doxorubicin quantification via HPLC-UV}

The amount of doxorubicin that was incorporated into the nanoparticles was determined by HPLC-UV (HPLC 1200 series, Agilent Technologies GmbH, Böblingen, Germany) using a LiChroCART $250 \times 4$ mm LiChrospher 100 RP 18 column (Merck KGaA, Darmstadt, Germany). The mobile phase was a mixture of water and acetonitrile (70:30) containing $0.1 \%$ trifluoroacetic acid [16]. In order to obtain symmetric peaks a gradient was used. In the first 6 min the percentage of A was reduced from $70 \%$ to $50 \%$. Subsequently within 2 min the amount of A was further decreased to $20 \%$ and then within another $2 \mathrm{~min}$ increased again to $70 \%$. These conditions were held for a final $5 \mathrm{~min}$, resulting in a total runtime of $15 \mathrm{~min}$. While using a flow rate of $0.8 \mathrm{~mL} / \mathrm{min}$, an elution time for doxorubicin of $t=7.5 \mathrm{~min}$ was achieved. The detection of doxorubicin was performed at a wavelength of $485 \mathrm{~nm}$ [34].

\section{Cell culture}

The neuroblastoma cell line UKF-NB-3, which harbours a MYCN amplification (a major indicator of high-risk disease and poor prognosis [35]), was established from a stage 4 neuroblastoma patient [20]. The UKF-NB-3 sub-lines adapted to growth in the presence of doxorubicin $20 \mathrm{ng} / \mathrm{mL}$ (UKF-NB$3^{\mathrm{r}} \mathrm{DOX}^{20}$ ) [20] or vincristine $1 \mathrm{ng} / \mathrm{mL}\left(\mathrm{UKF}-\mathrm{NB}-3^{\mathrm{r}} \mathrm{VCR}^{1}\right.$ ) were established by continuous exposure to step-wise increasing drug concentrations as previously described [20,36] and derived from the resistant cancer cell line (RCCL) collection [37].

All cells were propagated in Iscove's modified Dulbecco's medium (IMDM) supplemented with $10 \%$ foetal calf serum, $100 \mathrm{IU} / \mathrm{mL}$ penicillin and $100 \mu \mathrm{g} / \mathrm{mL}$ streptomycin at $37^{\circ} \mathrm{C}$. The drug-adapted sub-lines were continuously cultured in the presence of the indicated drug concentrations. The cells were 
routinely tested for mycoplasma contamination and authenticated by short tandem repeat profiling.

\section{Cell viability assay}

Cell viability was determined by 3-(4,5-dimethylthiazol-2-yl)2,5-diphenyltetrazolium bromide (MTT) assay modified after Mosman [38], as previously described [39]. $2 \times 10^{4}$ cells suspended in $100 \mu \mathrm{L}$ of cell culture medium were plated per well in 96-well plates and incubated in the presence of various doxorubicin concentrations (free or nanoparticle-encapsulated) for $120 \mathrm{~h}$. Where indicated, free or nanoparticle-encapsulated doxorubicin was combined with a fixed concentration of $1 \mu \mathrm{M}$ of the ABCB1 inhibitor zosuquidar. Then, $25 \mu \mathrm{L}$ of MTT solution $(2 \mathrm{mg} / \mathrm{mL}(\mathrm{w} / \mathrm{v})$ in PBS) was added per well, and the plates were incubated at $37{ }^{\circ} \mathrm{C}$ for an additional $4 \mathrm{~h}$. After this, the cells were lysed using $200 \mu \mathrm{L}$ of a buffer containing $20 \%$ (w/v) sodium dodecylsulfate and $50 \%(\mathrm{v} / \mathrm{v}) \mathrm{N}, \mathrm{N}$-dimethylformamide with the $\mathrm{pH}$ adjusted to 4.7 at $37{ }^{\circ} \mathrm{C}$ for $4 \mathrm{~h}$. The absorbance was determined at $570 \mathrm{~nm}$ for each well using a 96-well multiscanner. After subtracting of the background absorption, the results are expressed as percentage viability relative to control cultures which received no drug. The drug concentrations that inhibited cell viability by $50 \%\left(\mathrm{IC}_{50}\right)$ were determined using CalcuSyn (Biosoft, Cambridge, UK).

\section{Statistical testing}

The results are expressed as the mean \pm standard deviation of at least three experiments. The Student's $t$-test was used for comparing two groups. Three and more groups were compared by ANOVA followed by the Student-Newman-Keuls test. $P$-values lower than 0.05 were considered to be significant.

\section{Supporting Information}

Doxorubicin $\mathrm{IC}_{50} \mathrm{~s}$ in neuroblastoma cells in the absence or presence of the ABCB1 inhibitor zosuquidar. Effects of doxorubicin applied as solution or incorporated into HSA nanoparticles on neuroblastoma cell viability. Effects of doxorubicin solution or doxorubicin HSA nanoparticles on neuroblastoma cells with or without zosuquidar.

\section{Supporting Information File 1}

Additional experimental details.

[https://www.beilstein-journals.org/bjnano/content/ supplementary/2190-4286-10-166-S1.pdf]

\section{Acknowledgements}

This work was supported by the Kent Cancer Trust, the Hilfe für krebskranke Kinder Frankfurt e.V., and the Frankfurter Stiftung für krebskranke Kinder.

\section{ORCID ${ }^{\circledR}$ iDs}

Mark N. Wass - https://orcid.org/0000-0001-5428-6479

Klaus Langer - https://orcid.org/0000-0002-0203-7478

Martin Michaelis - https://orcid.org/0000-0002-5710-5888

\section{Preprint}

A non-peer-reviewed version of this article has been previously published as a preprint doi:10.1101/655662

\section{References}

1. International Agency for Research on Cancer. Cancer Fact Sheets. http://globocan.iarc.fr/Pages/fact_sheets_cancer.aspx (accessed Sept 10, 2018).

2. Holohan, C.; Van Schaeybroeck, S.; Longley, D. B.; Johnston, P. G. Nat. Rev. Cancer 2013, 13, 714-726. doi:10.1038/nrc3599

3. Steeg, P. S. Nat. Rev. Cancer 2016, 16, 201-218. doi:10.1038/nrc.2016.25

4. Siegel, R. L.; Miller, K. D.; Jemal, A. Ca-Cancer J. Clin. 2018, 68, 7-30 doi:10.3322/caac.21442

5. Robey, R. W.; Pluchino, K. M.; Hall, M. D.; Fojo, A. T.; Bates, S. E.; Gottesman, M. M. Nat. Rev. Cancer 2018, 18, 452-464. doi:10.1038/s41568-018-0005-8

6. Bar-Zeev, M.; Livney, Y. D.; Assaraf, Y. G. Drug Resist. Updates 2017, 31, 15-30. doi:10.1016/j.drup.2017.05.002

7. Thierry, A. R.; Vigé, D.; Coughlin, S. S.; Belli, J. A.; Dritschilo, A.; Rahman, A. FASEB J. 1993, 7, 572-579. doi:10.1096/fasebj.7.6.8097173

8. Bennis, S.; Chapey, C.; Robert, J.; Couvreur, P. Eur. J. Cancer 1994, 30, 89-93. doi:10.1016/s0959-8049(05)80025-5

9. Wong, H. L.; Bendayan, R.; Rauth, A. M.; Xue, H. Y.; Babakhanian, K.; Wu, X. Y. J. Pharmacol. Exp. Ther. 2006, 317, 1372-1381. doi:10.1124/jpet.106.101154

10. Prados, J.; Melguizo, C.; Ortiz, R.; Velez, C.; Alvarez, P. J.; Arias, J. L.; Ruiz, M. A.; Gallardo, V.; Aranega, A. Anti-Cancer Agents Med. Chem. 2012, 12, 1058-1070. doi:10.2174/187152012803529646

11. Oliveira, M. S.; Aryasomayajula, B.; Pattni, B.; Mussi, S. V.; Ferreira, L. A. M.; Torchilin, V. P. Int. J. Pharm. 2016, 512, 292-300. doi:10.1016/j.jpharm.2016.08.049

12. Maiti, C.; Parida, S.; Kayal, S.; Maiti, S.; Mandal, M.; Dhara, D. ACS Appl. Mater. Interfaces 2018, 10, 5318-5330. doi:10.1021/acsami.7b18245

13. Weber, C.; Kreuter, J.; Langer, K. Int. J. Pharm. 2000, 196, 197-200. doi:10.1016/s0378-5173(99)00420-2

14. Langer, K.; Balthasar, S.; Vogel, V.; Dinauer, N.; von Briesen, H.; Schubert, D. Int. J. Pharm. 2003, 257, 169-180. doi:10.1016/s0378-5173(03)00134-0

15. Langer, K.; Anhorn, M. G.; Steinhauser, I.; Dreis, S.; Celebi, D.; Schrickel, N.; Faust, S.; Vogel, V. Int. J. Pharm. 2008, 347, 109-117. doi:10.1016/j.ijpharm.2007.06.028

16. Dreis, S.; Rothweiler, F.; Michaelis, M.; Cinatl, J., Jr.; Kreuter, J.; Langer, K. Int. J. Pharm. 2007, 341, 207-214. doi:10.1016/j.ijpharm.2007.03.036

17. von Storp, B.; Engel, A.; Boeker, A.; Ploeger, M.; Langer, K. J. Microencapsulation 2012, 29, 138-146. doi:10.3109/02652048.2011.635218 
18. Ferrer, R.; Mateu, X.; Maseda, E.; Yébenes, J. C.; Aldecoa, C.; De Haro, C.; Ruiz-Rodriguez, J. C.; Garnacho-Montero, J. Expert Rev. Clin. Pharmacol. 2018, 11, 125-137. doi:10.1080/17512433.2018.1412827

19. Elzoghby, A. O.; Samy, W. M.; Elgindy, N. A. J. Controlled Release 2012, 157, 168-182. doi:10.1016/j.jconrel.2011.07.031

20. Kotchetkov, R.; Driever, P.; Cinatl, J.; Michaelis, M.; Karaskova, J.; Blaheta, R.; Squire, J.; Von Deimling, A.; Moog, J.; Cinatl, J., Jr. Int. J. Oncol. 2005, 27, 1029-1037. doi:10.3892/ijo.27.4.1029

21. Szakács, G.; Paterson, J. K.; Ludwig, J. A.; Booth-Genthe, C.; Gottesman, M. M. Nat. Rev. Drug Discovery 2006, 5, 219-234. doi:10.1038/nrd1984

22. Michaelis, M.; Rothweiler, F.; Klassert, D.; von Deimling, A.; Weber, K.; Fehse, B.; Kammerer, B.; Doerr, H. W.; Cinatl, J., Jr. Cancer Res. 2009, 69, 416-421. doi:10.1158/0008-5472.can-08-1856

23. Michaelis, M.; Rothweiler, F.; Löschmann, N.; Sharifi, M.; Ghafourian, T.; Cinatl, J., Jr. Oncotarget 2015, 6, 17605-17620. doi:10.18632/oncotarget.2889

24. Carter, D. C.; Ho, J. X. Adv. Protein Chem. 1994, 45, 153-203. doi:10.1016/s0065-3233(08)60640-3

25. Kavallaris, M. Nat. Rev. Cancer 2010, 10, 194-204. doi:10.1038/nrc2803

26. Wijdeven, R. H.; Pang, B.; Assaraf, Y. G.; Neefjes, J. Drug Resist. Updates 2016, 28, 65-81. doi:10.1016/j.drup.2016.07.001

27. Wischke, C.; Schwendeman, S. P. Int. J. Pharm. 2008, 364, 298-327. doi:10.1016/j.ijpharm.2008.04.042

28. Tyler, B.; Gullotti, D.; Mangraviti, A.; Utsuki, T.; Brem, H. Adv. Drug Delivery Rev. 2016, 107, 163-175. doi:10.1016/j.addr.2016.06.018

29. Pieper, S.; Onafuye, H.; Mulac, D.; Cinatl, J., Jr.; Wass, M. N.; Michaelis, M.; Langer, K. bioRxiv, Pharmacol. Toxicol. 2018. doi:10.1101/403923

30. Merlot, A. M.; Kalinowski, D. S.; Richardson, D. R. Front. Physiol. 2014, 5, 299. doi:10.3389/fphys.2014.00299

31. Hyun, H.; Park, J.; Willis, K.; Park, J. E.; Lyle, L. T.; Lee, W.; Yeo, Y. Biomaterials 2018, 180, 206-224. doi:10.1016/j.biomaterials.2018.07.024

32. Brufsky, A. Exp. Hematol. Oncol. 2017, 6, 7. doi:10.1186/s40164-017-0066-5

33. Vallo, S.; Köpp, R.; Michaelis, M.; Rothweiler, F.; Bartsch, G.; Brandt, M. P.; Gust, K. M.; Wezel, F.; Blaheta, R. A.; Haferkamp, A.; Cinatl, J., Jr. Oncol. Lett. 2017, 13, 4085-4092. doi:10.3892/ol.2017.5986

34. Sanson, C.; Schatz, C.; Le Meins, J.-F.; Soum, A.; Thévenot, J.; Garanger, E.; Lecommandoux, S. J. Controlled Release 2010, 147, 428-435. doi:10.1016/j.jconrel.2010.07.123

35. Fletcher, J. I.; Ziegler, D. S.; Trahair, T. N.; Marshall, G. M.; Haber, M.; Norris, M. D. Nat. Rev. Cancer 2018, 18, 389-400. doi:10.1038/s41568-018-0003-x

36. Michaelis, M.; Rothweiler, F.; Barth, S.; Cinatl, J.; van Rikxoort, M.; Löschmann, N.; Voges, Y.; Breitling, R.; von Deimling, A.; Rödel, F.; Weber, K.; Fehse, B.; Mack, E.; Stiewe, T.; Doerr, H. W.; Speidel, D.; Cinatl, J., Jr. Cell Death Dis. 2011, 2, e243. doi:10.1038/cddis.2011.129

37. University of Kent. The Resistant Cancer Cell Line (RCCL) collection. https://research.kent.ac.uk/industrial-biotechnology-centre/the-resistant -cancer-cell-line-rccl-collection/ (accessed Oct 19, 2018).

38. Mosmann, T. J. Immunol. Methods 1983, 65, 55-63. doi:10.1016/0022-1759(83)90303-4
39. Michaelis, M.; Matousek, J.; Vogel, J.-U.; Slavik, T.; Langer, K.; Cinatl, J.; Kreuter, J.; Schwabe, D.; Cinatl, J., Jr. Anti-Cancer Drugs 2000, 11, 369-376. doi:10.1097/00001813-200006000-00007

\section{License and Terms}

This is an Open Access article under the terms of the Creative Commons Attribution License (http://creativecommons.org/licenses/by/4.0). Please note that the reuse, redistribution and reproduction in particular requires that the authors and source are credited.

The license is subject to the Beilstein Journal of Nanotechnology terms and conditions:

(https://www.beilstein-journals.org/bjnano)

The definitive version of this article is the electronic one which can be found at: doi:10.3762/bjnano. 10.166 